Article

\title{
Cloning and Characterization of ThSHRs and ThSCR Transcription Factors in Taxodium Hybrid 'Zhongshanshan 406'
}

\author{
Zhiquan Wang ${ }^{1,2} \mathbb{D}^{1}$, Yunlong Yin ${ }^{1,2}$, Jianfeng Hua ${ }^{2}$, Wencai Fan ${ }^{2}$, Chaoguang $\mathrm{Yu}^{2}$, Lei Xuan ${ }^{2, *}$ \\ and Fangyuan $\mathrm{Yu}^{1}$,* \\ 1 Collaborative Innovation Center of Sustainable Forestry in Southern China, College of Forest Sciences, \\ Nanjing Forestry University, Nanjing 210037, China; zhiquanjiejie@163.com (Z.W.); yiny1066@sina.com (Y.Y.) \\ 2 Institute of Botany, Jiangsu Province and Chinese Academy of Sciences, Nanjing 210014, China; \\ jfhua2009@gmail.com (J.H.); fanfan461@sina.com (W.F.); yuchaoguang888@sina.com (C.Y.) \\ * Correspondence: 13851991791@163.com (L.X.); f yyu@njfu.com.cn (F.Y.); \\ Tel.: +86-25-8434-7096 (L.X.); +86-25-8542-7403 (F.Y.)
}

Academic Editor: Silvio Salvi

Received: 27 May 2017; Accepted: 17 July 2017; Published: 20 July 2017

\begin{abstract}
Among the GRAS family of transcription factors, SHORT ROOT (SHR) and SCARECROW (SCR) are key regulators of the formation of root tissues. In this study, we isolated and characterized two genes encoding SHR proteins and one gene encoding an SCR protein: ThSHR1 (Accession Number MF045148), ThSHR2 (Accession Number MF045149) and ThSCR (Accession Number MF045152) in the adventitious roots of Taxodium hybrid 'Zhongshanshan'. Gene structure analysis indicated that ThSHR1, ThSHR2 and ThSCR are all intron free. Multiple protein sequence alignments showed that each of the corresponding proteins, ThSHR1, ThSHR2 and ThSCR, contained five well-conserved domains: leucine heptad repeat I (LHRI), the VHIID motif, leucine heptad repeat II (LHR II), the PFYRE motif, and the SAW motif. The phylogenetic analysis indicated that ThSCR was positioned in the SCR clade with the SCR proteins from eight other species, while ThSHR1 and ThSHR2 were positioned in the SHR clade with the SHR proteins from six other species. Temporal expression patterns of these genes were profiled during the process of adventitious root development on stem cuttings. Whereas expression of both ThSHR2 and ThSCR increased up to primary root formation before declining, that of ThSHR1 increased steadily throughout adventitious root formation. Subcellular localization studies in transgenic poplar protoplasts revealed that ThSHR1, ThSHR2 and ThSCR were localized in the nucleus. Collectively, these results suggest that the three genes encode Taxodium GRAS family transcription factors, and the findings contribute to improving our understanding of the expression and function of SHR and SCR during adventitious root production, which may then be manipulated to achieve high rates of asexual propagation of valuable tree species.
\end{abstract}

Keywords: SHORT-ROOT; SCARECROW; adventitious root development; Taxodium

\section{Introduction}

Rapid increases in atmospheric $\mathrm{CO}_{2}$ concentration have resulted in climate change, such as global warming [1]. Afforestation and reforestation are recognized as economically efficient ways of ameliorating the problems associated with elevated atmospheric $\mathrm{CO}_{2}$ by increasing the extent of the forest carbon sink. The most widely-used method of vegetative propagation in forestry is stem cuttings from elite genotypes. The process of adventitious root formation and development, which is crucial to stem cutting, can be distinguished into three phases, induction, initiation and expression, 
according to previous reports [2]. However, for many ecologically and economically important tree species the inability of stem cuttings to form adventitious roots hinders the development of large-scale plantations [3]. In response to this problem, a number of studies on important tree species, investigating the process of adventitious root formation at the physiological, anatomical, biochemical and molecular levels, have been undertaken to overcome these problems [4,5]. Identification and characterization of the genes controlling adventitious root formation are essential to understanding and potentially manipulating this phenomenon, in order to propagate more tree species from cuttings.

The primary root and lateral roots build the adventitious root system jointly. SHORT ROOT (SHR) and SCARECROW (SCR) are part of the GRAS family of transcription factors, named after the first three members: GIBBERELLIC-ACID INSENSITIVE (GAI), REPRESSOR of GAI (RGA) and SCARECROW (SCR) and are key regulators of primary root stem cell definition and maintenance, and of radial patterning [6-8]. During, Arabidopsis development the intercellular movement of SHR and the subsequent interaction with its downstream target gene $S C R$ control root patterning and cell fate specification [9]. In the Arabidopsis root, SHR is expressed in the stele, then SHR moves into the adjacent cell layer to upregulate $S C R$ transcription and control endodermis specification [10-12]. In comparison, $S C R$ is expressed in the root cortex-endodermis initial cell and the endodermis of Arabidopsis root. The SCR protein, in turn, binds to its own promoter in the presence of SHR and JACKDAW (JKD)/IDD10, and they together regulate quiescent center markers and microRNAs involved in primary root vascular differentiation [13-15]. Loss-of-function mutations in SHR or SCR result in abnormal root development, indicating that SHR and SCR are essential for regulation of the asymmetric cell divisions which generate the root ground tissue; shr mutant roots lack the endodermis layer while the root ground tissue of $s c r$ mutants has a mixed cortex/endodermis identity $[5,16,17]$.

Despite the SHR/SCR network in the root system being investigated in detail in herbaceous model plants [12,18], there is no report of the isolation and characterization of the SHR and SCR genes in the adventitious roots of Taxodium hybrid 'Zhongshanshan'. T. 'Zhongshanshan' are interspecies hybrid clones generated from three Taxodium species, Taxodium mucronatum, Taxodium distichum, and Taxodium ascendens, and have been widely used as timber trees in river network areas, as windbreak trees in coastal areas, and as landscape trees in urban areas because of their extreme tolerance to a number of abiotic stresses, such as high salinity and waterlogging [19,20]. In this study, we identified two genes encoding SHR proteins (ThSHR1 and ThSHR2) and one gene encoding an SCR protein (ThSCR) from Taxodium hybrid 'Zhongshanshan 406', and characterized the genes with respect to their gene structures, sequence similarities, expression patterns and subcellular localizations.

\section{Materials and Methods}

\subsection{Plant Growth Conditions}

Softwood cuttings of Taxodium hybrid 'Zhongshanshan 406' (T. mucronatum $\circ \times$ T. distichum $0^{\text {') }}$ were collected from the Institute of Botany, Jiangsu Province \& Chinese Academy of Sciences, Nanjing, China. In July 2016, about 600 one-year old healthy softwood cuttings were selected for the experiment from the tops of mother seedlings less than 8-years old. Each cutting was then cut into a length of about $15 \mathrm{~cm}$, and one-third to one-half of the leaves on each cutting were removed to prevent excessive moisture loss. Then, $1000 \mathrm{mg} / \mathrm{L}$ carbendazim was used to thoroughly spray the seedbed (containing moistened perlite and peat soil with organic matter, 2:1) in a ventilated greenhouse under normal growth conditions (approximately $30^{\circ} \mathrm{C}$ ), in a photoperiod of $10 / 14 \mathrm{~h}$ of light/dark, to prevent mildew and other fungal attacks. After seedbed treatment, the cuttings were planted individually in the seedbed. The relative humidity was set at $70-80 \%$.

Based on the three phases of adventitious root formation and development, induction, initiation and expression, samples of cuttings were taken at two significant time points based on apparent morphological changes. The first time point (S1) was the initial formation of callus, the second (S2) was the time point when the primary root formed. And another time point, the lateral root formed 
period(S3), was added. The control time point (S0) was taken at 0 day, with the cuttings stored immediately after excision. The basal stem tissue $(0.2-0.5 \mathrm{~cm})$ (at S0 and S1) and the root tissue (at S2 and S3) of the cuttings were sampled for analysis at these four specific time points (Figure 1), at which the tissues were frozen immediately in liquid nitrogen and stored at $-80{ }^{\circ} \mathrm{C}$ until needed for extraction of RNA and DNA. Three independent biological replicates, each consisting of 20 randomly-selected stem cuttings, were taken at each time point.

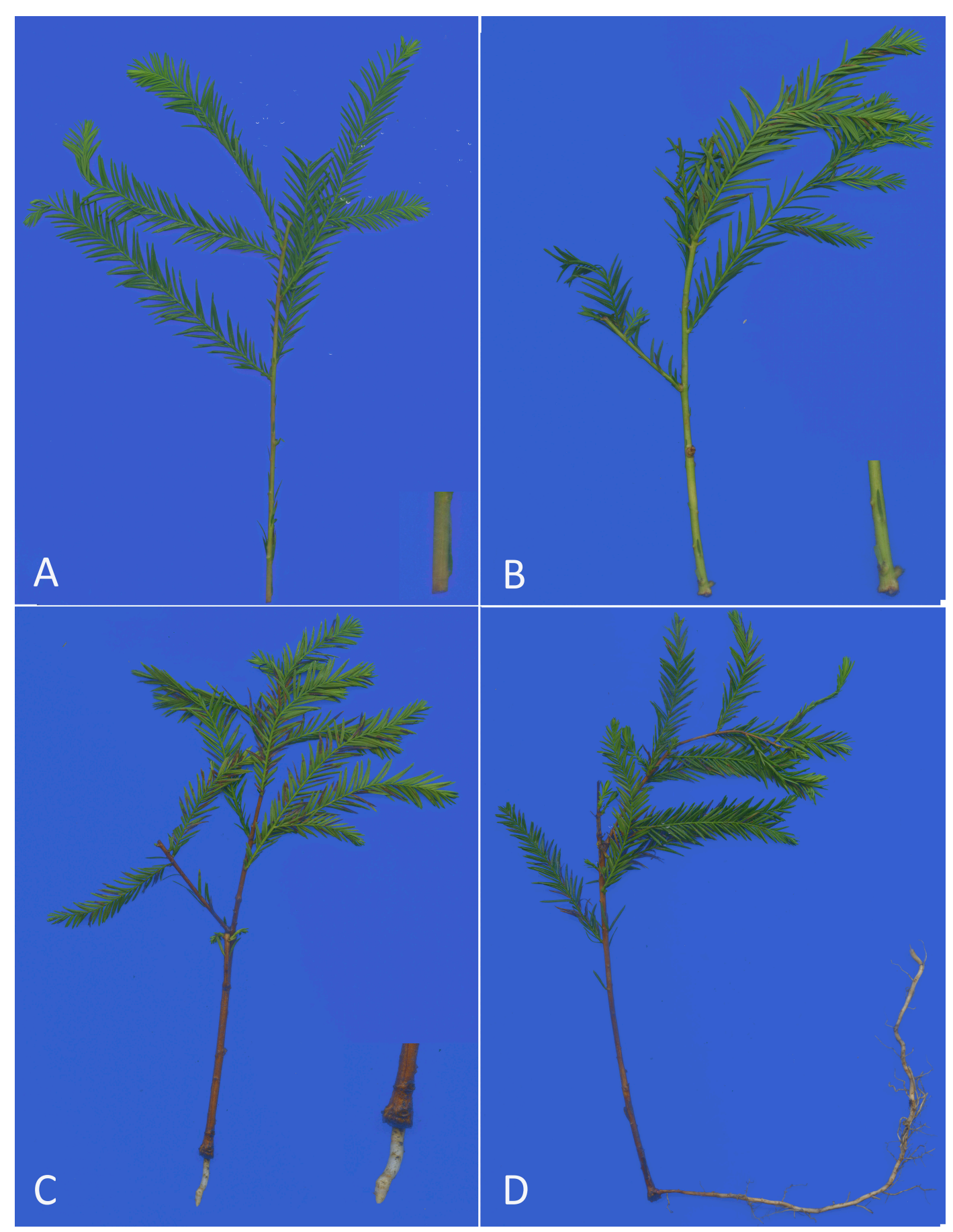

Figure 1. Adventitious root formation in Taxodium hybrid 'Zhongshanshan 406' at different developmental time points: (A) the control time point (S0); (B) the initial formation of callus (S1); (C) the formation of primary root (S2); and (D) lateral root systems (S3). 
MS agar medium was selected to cultivate the elite clone of hybrid poplar (Populus deltoides $\times$ Populus euramericana $\mathrm{cv}$. 'Nanlin895') for protoplast transfection under light intensity of $350 \mu \mathrm{mol} \cdot \mathrm{m}^{-2} \cdot \mathrm{s}^{-1}$ in the photoperiod of $16 / 8 \mathrm{~h}$ of day/night temperatures of $25 / 18{ }^{\circ} \mathrm{C}$. Newly-expanded young leaves of 6-week-old plants were used for protoplast isolation.

\subsection{Gene Cloning}

Total RNA was extracted using the RNAprep Pure Plant Kit (Polysaccharides- \& Polyphenolics-rich) (Tiangen, Beijing, China) and was treated with RNase-free DNase I (Qiagen, Dusseldorf, Germany). RNA concentration was measured with a spectrophotometer (NanoDrop2000, Thermo Scientific, Waltham, MA, USA) at 260 and $280 \mathrm{~nm}$, and samples with the 260/280 nm ratio within the range $1.80-2.20$ were used. RNA integrity was also checked using $2 \%$ agarose gels. The DNase-treated RNA of adventitious roots (S2 and S3) was used for rapid amplification of cDNA ends (RACE). Based on sequences provided from the previously-obtained transcriptomics data (unpublished data), fragment sequences, from the middle region, were PCR-amplified and sequenced $[19,20]$. Then, based on the fragment sequences, the nested primers were designed using the Oligo 6.0 software (Molecular Biology Insights, Colorado Springs, CO, USA) to amplify the full-length sequences with the 3-Full RACE Core Set Kit (TaKaRa, Otsu, Japan) and SMARTer RACE 5' /3'Kit (Clontech, Palo Alto, CA, USA), according to the manufacturer's instructions. The PCR products were recovered and cloned into pMD19-T vectors (TaKaRa), finally transformed into competent cells of the Escherichia coli strain DH5 $\alpha$, and sent to the sequencing facility. By comparing and aligning the sequences of $3^{\prime}$-RACE and 5'-RACE and the middle region products, the full-length cDNA sequences of the two ThSHRs and of ThSCR were obtained. The predicted open reading frames (ORFs) were then amplified by PCR, and were verified by sequencing. Genomic DNA was extracted using a Plant Genomic DNA Extraction Kit (BioTeke, Beijing, China). Genomic DNA sequences of the ORFs of the above genes were amplified from RNase-treated DNA using the same primers, and were verified by sequencing. The sequences of the primers are listed in Table 1.

Table 1. Primer sequences of the genes for rapid amplification of cDNA ends (RACE), full-length open reading frames (ORFs) and real-time PCR (qRT-PCR).

\begin{tabular}{|c|c|c|}
\hline Primer-ID & Forward PCR Primer $\left(5^{\prime}-3^{\prime}\right)$ & Reverse PCR Primer $\left(5^{\prime}-3^{\prime}\right)$ \\
\hline ThSHR1_3OUTER & TGTGTGAGCGCCGTGAGAAGGCTGTGCA & TACCGTCGTTCCACTAGTGATTT \\
\hline ThSHR1_3INNER & TTTTGCAGTTTTTTGTAAGTCCTG & CGCGGATCCTCCACTAGTGATTTCACTATAGG \\
\hline ThSHR1_5OUTER & СТAATACGACTCACTATAGGGCAAGCAGTGGTATCAACGCAGAGT & ATCCATAGAAGATGCTGCATTCGC \\
\hline ThSHR1_5INNER & CTAATACGACTCACTATAGGGC & GTTGGTGGTGGTATTGGGCTTCTC \\
\hline ThSHR1_ORF & ATGGATAGATTGTTTACCTCCAGCATA & TTACAAGCAAGGCTTCCAGGCGG \\
\hline ThSHR1_qRT-PCR & TACCCGCGACCTTCAGTGAC & CAGGCGGAAGCCCAAATGAC \\
\hline ThSHR2_3OUTER & TTCTGTTATGAAGGAAATAGGGCA & TACCGTCGTTCCACTAGTGATTT \\
\hline ThSHR2_3INNER & TCAGCCTGTGTTTCGTTTCGCTTGA & CGCGGATCCTCCACTAGTGATTTCACTATAGG \\
\hline ThSHR2_5OUTER & СTAATACGACTCACTATAGGGCAAGCAGTGGTATCAACGCAGAGT & CTGAACTCAAACGGCACTCCСATAA \\
\hline ThSHR2_5INNER & CTAATACGACTCACTATAGGGC & AGGGACTAGCCTCCTGGAATTTCAG \\
\hline ThSHR2_ORF & ATGTCATATAGTTTTTATACCCATTC & CTATTTAGGTTTCCAGGCAGAAG \\
\hline ThSHR2_qRT-PCR & ACTCAGTGGCCCATGCTGTT & AACGGCACTCCCATAAGCCT \\
\hline ThSCR_3OUTER & ATCCTGATGACAGCATAGAAAGACA & TACCGTCGTTCCACTAGTGATTT \\
\hline ThSCR_3INNER & TGGAACTCTCAAACTTGGCTGGAAAGAC & CGCGGATCCTCCACTAGTGATTTCACTATAGG \\
\hline ThSCR_5OUTER & CTAATACGACTCACTATAGGGCAAGCAGTGGTATCAACGCAGAGT & ACATTGGAGTAGAAGATCCAGAAGG \\
\hline ThSCR_5INNER & CTAATACGACTCACTATAGGGC & TCCAGTTTGCAGCTCCAATTTCATC \\
\hline ThSCR_ORF & ATGACATTTGCTTCTTCTAAATTGTGTG & TTACAGTGCATCATGGGTGGGTGTC \\
\hline ThSCR_qRT-PCR & ATGGCCAGGGCTATTCCACA & CTGCCTGTTGCCTCCAATGC \\
\hline APRT_qRT-PCR & TCCACAGGTTCTTGAATCGCT & TGACTTGAGCCTCATTCGCTC \\
\hline
\end{tabular}

\subsection{Sequence Analysis}

Online BLAST software was used to analyze the DNA and protein sequences (https://blast. ncbi.nlm.nih.gov/Blast.cgi). ORFs were predicted with the ORFfinder program (https://www.ncbi. nlm.nih.gov/orffinder/). Gene predictions were performed using the FGENESH program(http:// linux1.softberry.com/berry.phtml?topic=fgenesh\&group=programs\&subgroup=gfind). The theoretical isoelectric point $(\mathrm{pI})$, molecular weight $(\mathrm{MW})$ and amino acid composition of the proteins were predicted and calculated using Expasy Protparam (http://web.expasy.org/protparam/). 
Protein transmembrane structures, protein domain and signal peptide cleavage site analyses were performed using the TMHMM (http://www.cbs.dtu.dk/services/TMHMM/), PROSITE (http://prosite.expasy.org/) and SignalP online tools (http://www.cbs.dtu.dk/services/SignalP/), respectively. Secondary structures of deduced amino acid sequences were predicted by the GOR IV secondary structure prediction method (https://npsa-prabi.ibcp.fr/cgi-bin/npsa_automat.pl?page= npsa_gor4.html). The deduced protein sequences of ThSHR1, ThSHR2 and ThSCR were aligned with other plant SHR and SCR sequences using the BioEdit 2.6 software (http:/ /www.mbio.ncsu.edu/ BioEdit/bioedit.html). The putative nuclear localization signals were predicted with NLStradamus (http://www.moseslab.csb.utoronto.ca/NLStradamus/). The phylogenetic tree was constructed using MEGA 5.0 with Maximum Likelihood method and 1000 bootstraps [21].

\subsection{Real Time PCR Analysis of ThSHRs and ThSCR}

The complementary DNA (cDNA) was synthesized for real-time PCR based on $1 \mu \mathrm{g}$ DNase-treated RNA using PrineScript@RTase (200 U) system (TaKaRa), according to the manufacturer's instructions. Primers for the reference gene adenine phosphoribosyl transferase (APRT) and the three target genes were designed using the Oligo 6.0 software with the following parameters: melting temperatures of $58-62{ }^{\circ} \mathrm{C}$, primer lengths of $19-25 \mathrm{bp}$, GC content of $40-60 \%$ and amplicon lengths of $80-210 \mathrm{bp}$ (Table 1) [22]. Real-time PCR was conducted in 96-well plates and performed on the Analitik Jena qTOWER2.2 PCR System (Biometra, Gottingen, Germany) using the following cycling conditions: $50{ }^{\circ} \mathrm{C}$ for $2 \mathrm{~min}, 95^{\circ} \mathrm{C}$ for $10 \mathrm{~min}$, and 40 cycles of $95^{\circ} \mathrm{C}$ for $15 \mathrm{~s}, 60^{\circ} \mathrm{C}$ for $1 \mathrm{~min}$ followed by a melting curve analysis by heating the PCR products from 60 to $95^{\circ} \mathrm{C}$. Each reaction mix contained $2 \mu \mathrm{L}$ cDNA of previously diluted cDNA (1:3), $10 \mu \mathrm{L}$ FastStart Universal SYBR Green Master (ROX, Roche Applied Science, Mannheim, Germany), $6.8 \mu \mathrm{L}$ RNase-free water, and 6 pmol of each primer, for a final volume of $20 \mu \mathrm{L}$. A no-template control was also included in each run for each gene. Each real-time PCR analysis was performed in triplicate. The calculations of relative expression levels between the target and reference genes were performed using the delta-Ct method $[23,24]$.

\subsection{Protoplast Transfection}

In this study, plasmids were constructed by Gateway Technology (Invitrogen, Leek, The Netherlands), following the manufacturer's protocol. First, the ThSHR (or ThSCR) coding regions (without the stop codon) were cloned into the entry vector, pCR8/GW/TOPO (Invitrogen), with a simple TOPO cloning reaction. In order to perform the subcellular localization of tagged proteins, an LR clonase enzyme mix (Invitrogen) was used to transfer the insert from the entry vector into its destination vector, p2GWF7, with a green fluorescent protein (GFP) tag positioned at the C-terminal of the insert. The GFP fusion vectors (35S::ThSHR1-GFP,35S::ThSHR2-GFP, and 35S::ThSCR-GFP) generated were high-copy vectors, driven by the promoter of double $35 \mathrm{~S}$ cauliflower mosaic virus with ampicillin as the selectable bacterial marker. Protoplast isolation and polyethylene glycol-mediated transfection were performed using the method described by [25]. A BX51 fluorescence microscope (Olympus America, Center Valley, PA), equipped with $450-490 \mathrm{~nm} / 520-560 \mathrm{~nm}$ and $540-580 \mathrm{~nm} / 600-660 \mathrm{~nm}$ (excitation/emission) filters, was used to capture images.

\section{Results}

\subsection{Molecular Cloning and Sequence Analysisof ThSHR and ThSCR Genes}

Three target genes, termed ThSHR1, ThSHR2 and ThSCR, were successfully isolated and identified by $3^{\prime}$-RACE and $5^{\prime}$-RACE procedures. Comparisons of genomic and CDNA sequences showed that ThSHR1, ThSHR2 and ThSCR were all intron-free. The full-length sequence of ThSHR1 cDNA was $2069 \mathrm{bp}$, containing an ORF of $1455 \mathrm{bp}$, flanked by $332 \mathrm{bp}$ of $5^{\prime}$-untranslated region (UTR) and a $282 \mathrm{bp}$ 3'-UTR; ThSHR2 was $1515 \mathrm{bp}$ with an ORF of $1290 \mathrm{bp}$, flanked by 52 and $173 \mathrm{bp} 5^{\prime}$ - and 3'-UTRs, 
respectively; ThSCR was 1909 bp with an ORF of 1596 bp, flanked by 220 and 93 bp 5'- and 3'-UTRs, respectively (Table 2).

Genes ThSHR1, ThSHR2 and ThSCR encode polypeptides of 484, 429 and 531 amino acid residues, respectively. The GOR IV secondary structure prediction method was used to predict the secondary structures of these three proteins [26]. TheThSHR1, ThSHR2 and ThSCR proteins contained $41.32 \%$, $32.87 \%$ and $38.61 \%$ of alpha helices $(\mathrm{Hh})$, respectively; and correspondingly $14.46 \%, 19.11 \%$ and $17.33 \%$ of extended strands (Ee), respectively; and $44.21 \%, 48.02 \%$ and $44.07 \%$ of random coils (Cc), respectively; interestingly, the three proteins all contained zero beta turns (Tt) (Table 2). The Prosite reports suggested that the three proteins had a typical GRAS domain: amino acid residues 89-462 for ThSHR1 (score 51.621), 38-407 for ThSHR2 (score 50.775) and 138-505 for ThSCR (score 58.917) [27].

The protein sequences of ThSHR1, ThSHR2 and ThSCR were compared with Arabidopsis protein sequences in TAIR (AtSHR, At4g37650.1; AtSCR, At3g54220.1) and Populus protein sequences based on previous studies [28-31]. The result indicated that the two SHR proteins and one SCR protein belonged to the plant-specific GRAS family of transcription factors and exhibited considerable sequence similarity to one other throughout their carboxyl termini, including leucine heptad repeat I (LHRI), the VHIID motif, leucine heptad repeat II (LHRII), the PFYRE motif and the SAW motif (Figures 2 and 3). The putative nuclear localization signals were marked with red points in Figures 2 and 3.

Table 2. Features of the ThSHR1, ThSHR2 and ThSCR.

\begin{tabular}{|c|c|c|c|c|c|c|c|c|c|c|c|}
\hline \multirow[b]{2}{*}{ Gene_ID } & \multirow{2}{*}{$\begin{array}{c}\text { Full- } \\
\text { Length } \\
\text { cDNA(bp) }\end{array}$} & \multirow{2}{*}{$\begin{array}{l}5^{\prime}- \\
\text { UTR } \\
\text { (bp) }\end{array}$} & \multirow{2}{*}{$\begin{array}{c}3^{\prime}- \\
\text { UTR } \\
\text { (bp) }\end{array}$} & \multirow{2}{*}{$\begin{array}{l}\text { ORF } \\
\text { (bp) }\end{array}$} & \multicolumn{3}{|c|}{ Predicted Peptide } & \multicolumn{4}{|c|}{ Secondary Structure Prediction } \\
\hline & & & & & $\begin{array}{c}\text { MW } \\
\text { (kDa) }\end{array}$ & PI & GRAVY & $\begin{array}{l}\mathrm{Hh} \\
(\%)\end{array}$ & $\begin{array}{c}\text { Ee } \\
(\%)\end{array}$ & $\begin{array}{l}\mathrm{Tt} \\
(\%)\end{array}$ & $\begin{array}{c}\mathrm{Cc} \\
(\%)\end{array}$ \\
\hline ThSHR1 & 2069 & 332 & 282 & 1455 & 55.52 & 5.58 & -0.383 & 41.32 & 14.46 & 0.00 & 44.21 \\
\hline ThSHR2 & 1515 & 52 & 173 & 1290 & 48.80 & 5.26 & -0.256 & 32.87 & 19.11 & 0.00 & 48.02 \\
\hline ThSCR & 1909 & 220 & 93 & 1596 & 59.12 & 5.43 & -0.224 & 38.61 & 17.33 & 0.00 & 44.07 \\
\hline
\end{tabular}

UTR: untranslated region; ORF: open reading frame; MW: molecular weight; PI: isoelectric point; GRAVY: grand average of hydropathicity; Hh: alpha helices; Ee: extended strands; Tt: beta turns; Cc: random coils.

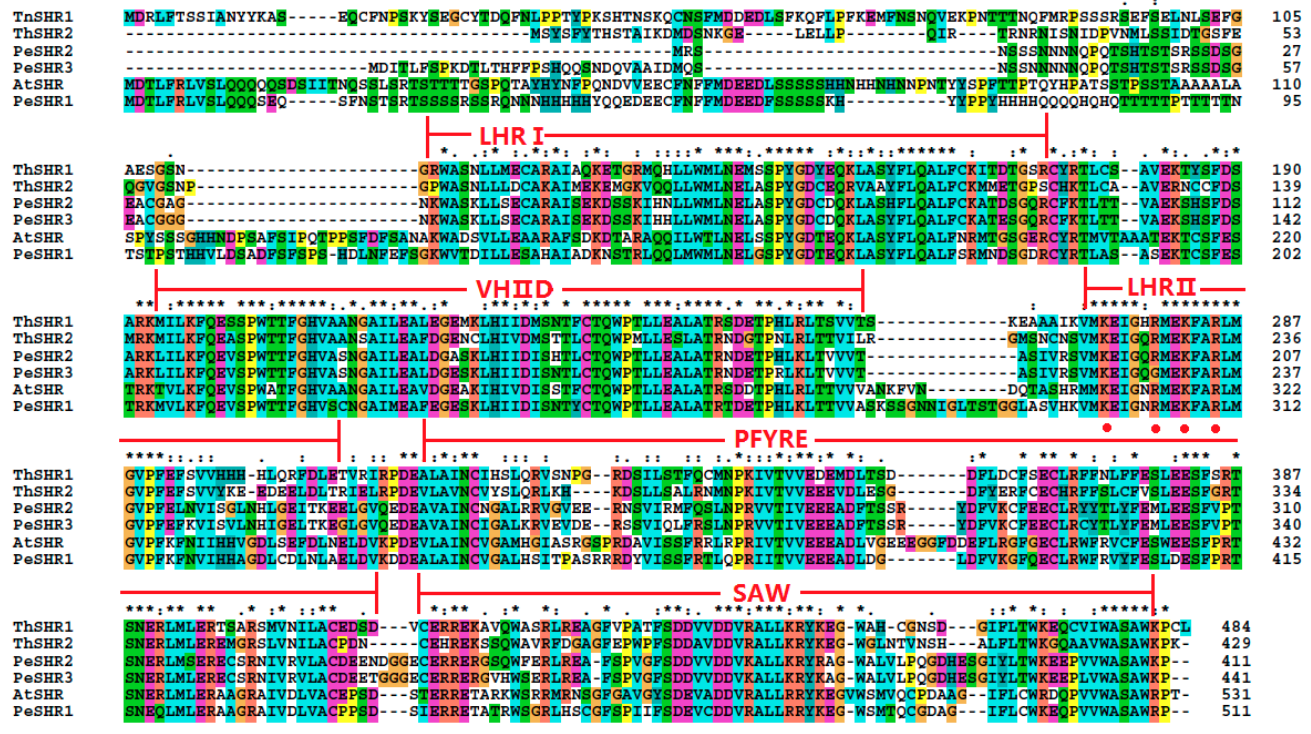

Figure 2. Alignment of the amino acid sequences of ThSHR1 and ThSHR2 with those of SHORT ROOT (SHR) of both Arabidopsis and Populus, using the ClustalW program. The highly-conserved region of the GRAS proteins can be divided into five recognizable motifs: the leucine heptad repeat I (LHR1), the VHIID motif, the leucine heptad repeat II (LHRII), the PFYRE motif and the SAW motif, as indicated above the sequence alignment. Amino acid position numbers are indicated to the right of the sequence. The putative nuclear localization signals were labeled with red points. The same below. 


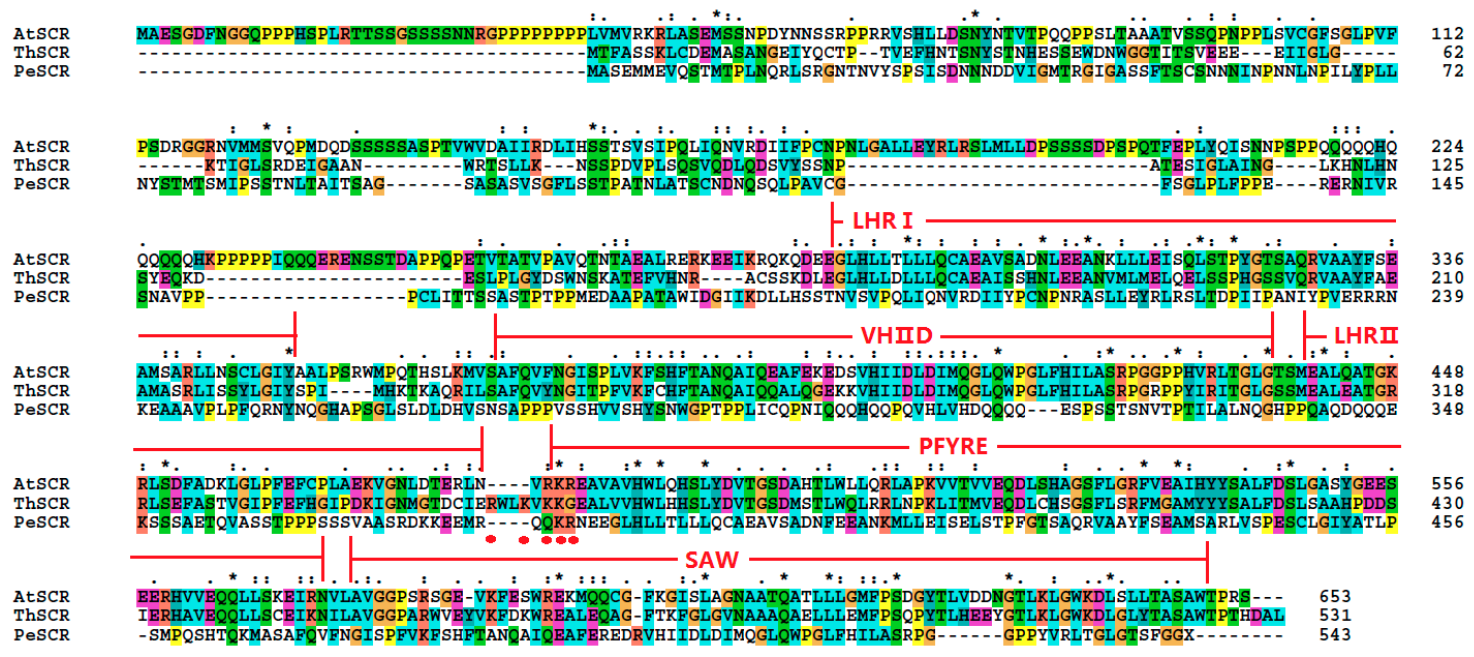

Figure 3. Alignment of the amino acid sequences of ThSCR with those of SCARECROW (SCR) of both Arabidopsis and Populus.

To understand the evolutionary relationship of GRAS proteins from different species, the amino acid sequences of ThSHR1, ThSHR2 and ThSCR (GRAS domains) were aligned with those of 48 full-length GRAS proteins, and an un-rooted neighbor-joining (NJ) phylogenetic tree was constructed using MEGA 5.0 software and the maximum likelihood method with 1000 replicate bootstrap testing. The GRAS protein family can be divided into several clades, which have been designated after one of their members or their common features [32]. In this study, the un-rooted NJ tree based on multiple sequence alignments showed that the 51 protein domains were clustered into eight distinct groups (Figure 4). The result showed that ThSHR1 and ThSHR2, most closely related to the conifer proteins PrSHR (from Pinus radiata) and PtSHR (from Pinus taeda), were positioned in the SHR clade; ThSCR was positioned in the SCR clade, and had an identity of 52\% with AtSCL23 (Figure 4). 


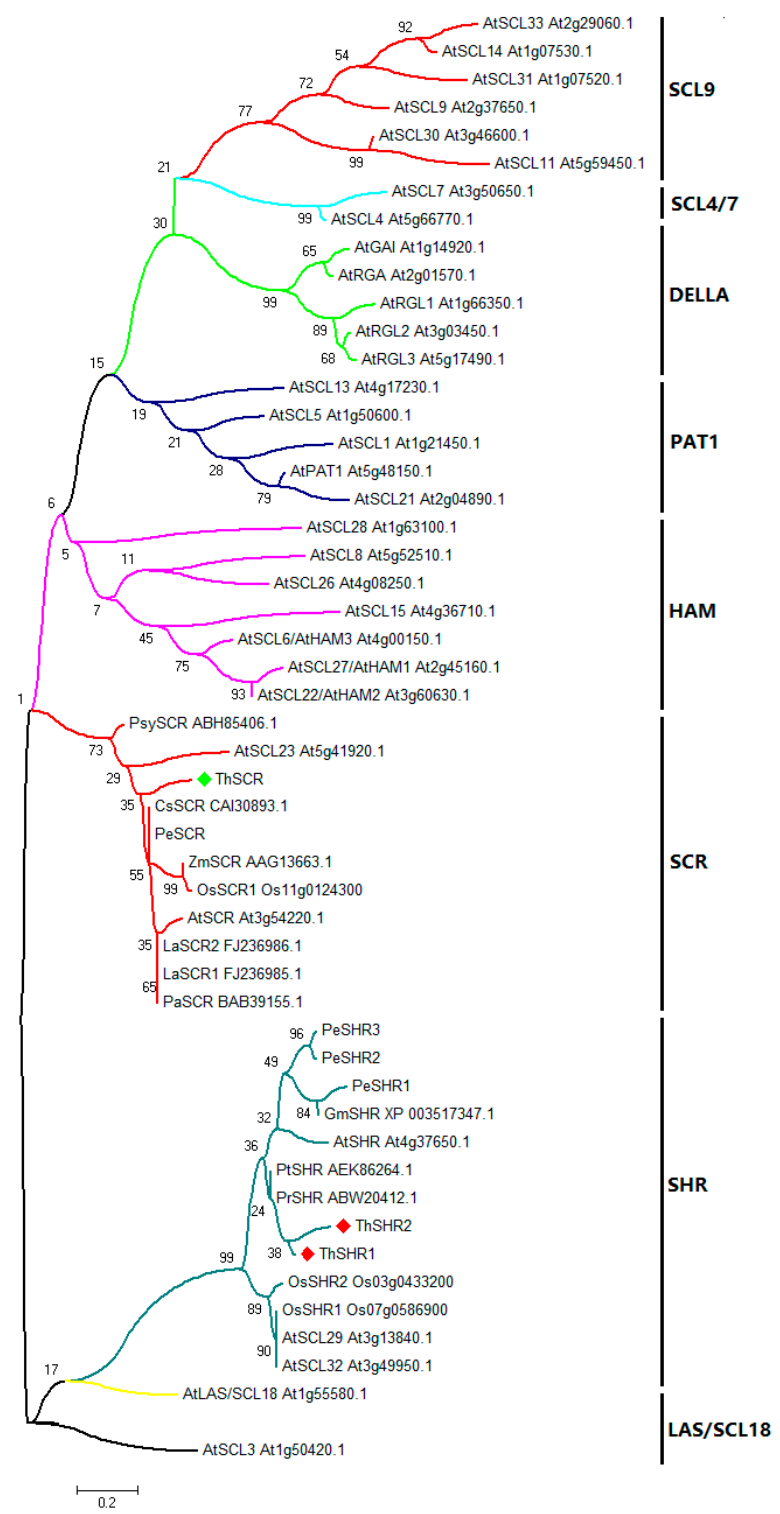

Figure 4. Phylogenetic analysis of the proteins of the GRAS gene family. The phylogenetic tree was constructed using MEGA 5.0 with the maximum likelihood method using 1000 replicate bootstrap tests. Numbers near to the nodes indicate bootstrap values obtained from 1000 replications. The 51 proteins were clustered into eight distinct groups: SCL9, PAT1, LAS/SCL18, SCL4/7, SHR, SCR, HAM and DELLA, as indicated. At, Arabidopsis thaliana; Os, Oryza sativa; Gm, Glycine max; Pe, Populus; Pr, Pinus radiata; Pt, Pinus taeda; La, Lupinus albus; Ps, Pisum sativum; Cs, Cucumis sativa; Zm, Zea mays; Psy, Pinus sylvestris.

\subsection{Expression of ThSHR and ThSCR}

To analyze the expression patterns of the ThSHR1, ThSHR2 and ThSCR genes in T. 'Zhongshanshan 406', we measured their transcript levels by real-time PCR at different developmental time points: the control time point (S0), the initial formation of callus(S1), the formation of primary root(S2) and lateral root systems(S3) (Figure 1). The gene expression patterns of ThSHR2 and ThSCR were similar over the period of adventitious root formation, with an apparent increase-decrease trend over time. The maximum expression of both genes occurred at S2, with the minimum expression at either S3 (ThSCR) or S0 (ThSHR2). In contrast with these two genes, the expression pattern of ThSHR1 showed a trend of increasing expression throughout the process of adventitious root formation (Figure 5). 

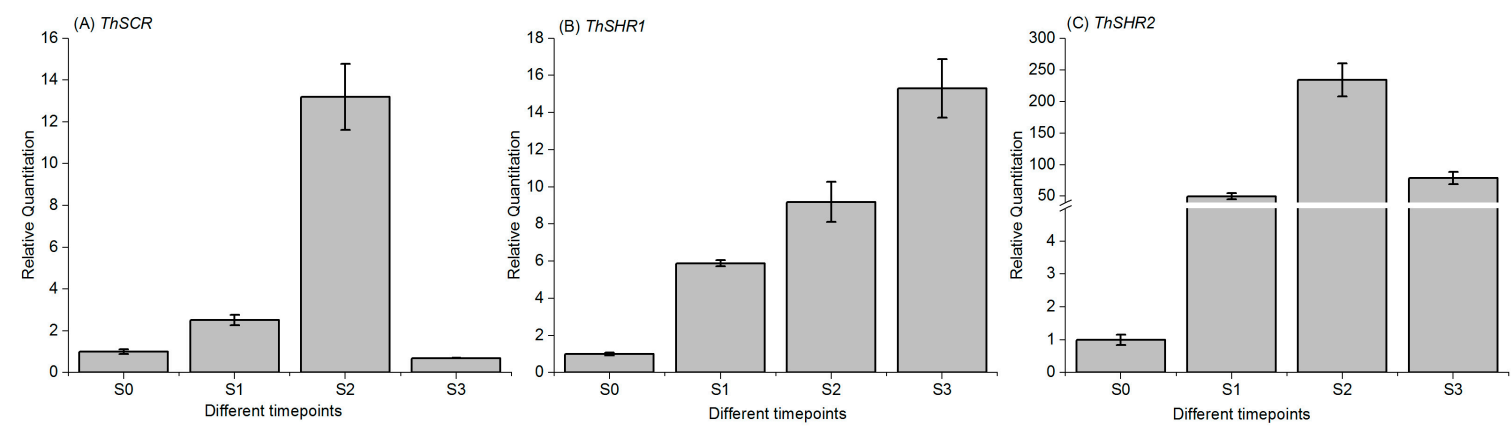

Figure 5. Temporal expression patterns of ThSHR1, ThSHR2 and ThSCR by real-time PCR. S0, S1, S2 and $\mathrm{S} 3$ are the tissues at 0 day, initial formation of callus, primary root and the lateral roots, respectively. For real-time PCR, adenine phosphoribosyl transferase (APRT) was used as the internal control, and the relative transcript levels were calculated using the comparative delta-Ct method. Error bars represent the standard deviation for each sample, based on three biological replicates (the averages of technical replicates).

\subsection{Subcellular Localization of ThSHR and ThSCR Proteins}

In order to probe the subcellular localizations of the ThSHR1, ThSHR2 and ThSCR proteins, the GFP-fusion vectors (35S::ThSHR1-GFP, 35S::ThSHR2-GFP and 35S::ThSCR-GFP) were transformed into Populus protoplasts under the control of the double $35 \mathrm{~S}$ cauliflower mosaic virus promoter. The cellular localization of the fusion protein was observed using confocal microscopy. All three proteins were located in the nucleus. As a positive control, the 35::GFP fusion protein was detected in the nucleus and cytoplasm of Populus protoplasts (Figure 6).
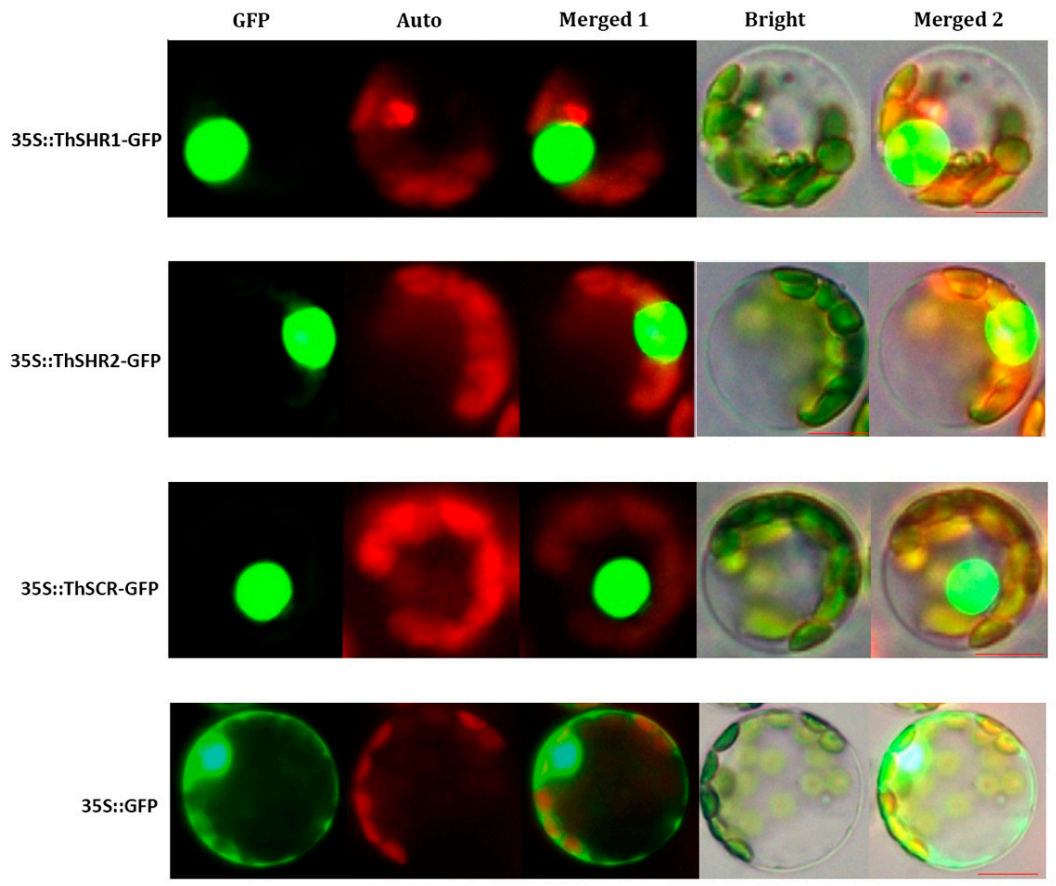

Figure 6. Subcellular localization of ThSHR1, ThSHR2 and ThSCR. Green fluorescent protein (GFP), chlorophyll auto fluorescence (Auto), merged1, bright and merged 2 images are shown. Scale bar: $5 \mu \mathrm{m}$. The 35::GFP fusion was used as the positive protein control, and was detected in the nucleus and cytoplasm of Populus protoplasts. 


\section{Discussion}

Transcription factors (TFs), also known as trans-acting factors, are located in the nuclei and exhibit a special interaction with cis-regulatory elements in the gene promoter regions. As a result, expression of a set of downstream functionally-related target genes are regulated in a spatio-temporal specific manner [33]. Hence, TFs are core components of gene regulation networks [33,34]. SHR and SCR belong to the plant-specific GRAS TF family $[32,35,36]$. It has been reported that SHR and SCR play key roles in formation of root ground tissue [6,37]. They are essential to regulate the asymmetric cell divisions, and thereby to generate the root ground tissue [38-40]. To date, a number of SHR and SCR genes involved in seedling root system have been studied systematically $[9,12,33]$. However, studies in SHR and SCR genes involving adventitious roots were few and far between.

In this study, two ThSHR and one ThSCR genes, which may be involved in adventitious root development of Taxodium hybrid 'Zhongshanshan', were isolated and characterized in the adventitious roots. The gene structure analysis indicated that these three genes were all intron-free. Similar results were obtained in Populus [31]. The Prosite reports have suggested that the three proteins had a typical GRAS domain, thereby confirming that the three proteins belong to the plant-specific GRAS family of TFs [35]. The result of multiple sequence alignments indicated thatThSHR1, ThSHR2 and ThSCR showed considerable protein sequence similarity with other SHRs and SCRs of Arabidopsis and Populus throughout their carboxyl termini, including the LHRI, VHIID, LHRII, PFYRE and SAW motifs. On the other hand, the three proteins showed variation in the length and amino acid sequence at the amino-termini. Such variation may reflect the ability of the proteins to bind to different promoter nucleotide sequences and accessory proteins [32]. In the previous study, Pysh hypothesized that the area encompassing the two LRRs and the VHIID motif may act as a DNA binding domain analogous to the bZIP protein-DNA interaction domain, with the LRRs mediating protein-protein interactions, and the VHIID motif mediating DNA-protein interactions [41]. So, we also hypothesized that the area encompassing the LHRI and the VHIID motif may act as a DNA binding domain analogous.

Meanwhile, the phylogenetic analysis of plant GRAS proteins indicates thatThSHR1, ThSHR2 and ThSCR showed highly similarity to other GRAS proteins. ThSCR was positioned in the SCR clade with SCR proteins from eight other species, including A. thaliana, O. sativa, Z. mays, Lupinus albus, Cucumis sativa, Populus, Pisum sativum and Pinus sylvestris. ThSHR1 and ThSHR2 were positioned in the SHR clade with SHR proteins from six other species. Interestingly, the two ThSHR proteins were most closely related to two Pinus species, also conifers like Taxodium. On the whole, the results of this study are consistent with previous reports [32]. Consistent with their role as transcriptional regulators, the ThSHR1-GFP, ThSHR2-GFP and ThSCR-GFP fusion proteins were located in the nucleus, similar to the situation in Populus cells [31]. Combined together, these features provide strong evidence that the three genes isolated here encode Taxodium GRAS family transcription factors. The difference of localization of SHR of Taxodium to that of Arabidopsis maybe due to species difference, and this could be further investigated in future studies in this area [42,43].

In studying the expression patterns, we measured their transcript levels by real-time PCR at different developmental time points: the control time point (S0), the initial formation of callus (S1), the formation of primary root (S2) and lateral root systems (S3) (Figure 1). In contrast to previous studies, we added a stage after expression (lateral root formed period (S3)) to explore the subsequent expression of genes [2]. Expression patterns of these three genes showed that expression of the genes ThSHR2 and ThSCR was highest at the period of S2, then began to decline, while that of gene ThSHR1 exhibited steady increases in expression throughout the process of adventitious root formation and development. The expression pattern of ThSHR1 is similar to the previous reports, while ThSHR2 and ThSCR are not exactly consistent with previous studies [31]. The possible reasons are differences in design and differences in species. This result revealed that the ThSHR1, ThSHR2 and ThSCR genes might be involved in the formation and development of adventitious roots. This finding contributes to improving our understanding of how genes SHR and SCR function in the development of adventitious root formation, and could result in advances in asexual propagation of normally recalcitrant species, 
such as the conifers. Future research is necessary to verify the roles of ThSHR and ThSCR by in situ hybridization, and their functions by genetic transformation. Elucidating whether these proteins interact with each other is also important.

Acknowledgments: This work was supported by the Innovation Project of Plant Germplasm Resources of Chinese Academy of Sciences (ZSZC-009), Jiangsu Agriculture Science and Technology Innovation Fund (CX(16)1005), the Program of Innovation Capacity Construction of Jiangsu Province (BM 2015019) and the Innovative Research Project for Postgraduates in Colleges of Jiangsu Province.

Author Contributions: W.Z. and Y.Y. conceived and designed the experiments; W.Z., H.J., F.W., and C.Y. performed the experiments; W.Z. analyzed the data; W.Z., X.L., and Y.F. wrote the paper.

Conflicts of Interest: The research was conducted in the absence of any commercial or financial relationships that could be construed as a potential conflict of interest. The founding sponsors had no role in the design of the study; in the collection, analyses, or interpretation of data; in the writing of the manuscript, and in the decision to publish the results.

\section{References}

1. Guo, F.; Song, Z.; Sullivan, L.; Wang, H.; Liu, X.; Wang, X.; Li, Z.; Zhao, Y. Enhancing phytolith carbon sequestration in rice ecosystems through basalt powder amendment. Sci. Bull. 2015, 60, 591-597. [CrossRef]

2. Porfírio, S.; da Silva, M.D.R.G.; Cabrita, M.J.; Azadi, P.; Peixe, A. Reviewing current knowledge on olive (Olea europaea L.) adventitious root formation. Sci. Hortic. 2016, 198, 207-226.

3. Xu, M.; Xie, W.; Huang, M. Two WUSCHEL-related HOMEOBOX genes, PeWOX11a and PeWOX11b, are involved in adventitious root formation of poplar. Physiol. Plant. 2015, 155, 446-456. [CrossRef] [PubMed]

4. Zhang, B.; Tong, C.; Yin, T.; Zhang, X.; Zhuge, Q.; Huang, M.; Wang, M.; Wu, R. Detection of quantitative trait loci influencing growth trajectories of adventitious roots in Populus using functional mapping. Tree Genet. Genomes 2009, 5, 539-552. [CrossRef]

5. Da Costa, C.T.; De Almeida, M.R.; Ruedell, C.M.; Schwambach, J.; Maraschin, F.D.S.; Fett-Neto, A.G. When stress and development go hand in hand: Main hormonal controls of adventitious rooting in cuttings. Front. Plant Sci. 2013, 4, 133. [CrossRef] [PubMed]

6. Di Laurenzio, L.; Wysocka-Diller, J.; Malamy, J.E.; Pysh, L.; Helariutta, Y.; Freshour, G.; Hahn, M.G.; Feldmann, K.A.; Benfey, P.N. The SCARECROW gene regulates an asymmetric cell division that is essential for generating the radial organization of the Arabidopsis root. Cell 1996, 86, 423-433. [CrossRef]

7. Sabatini, S.; Heidstra, R.; Wildwater, M.; Scheres, B. SCARECROW is involved in positioning the stem cell niche in the Arabidopsis root meristem. Genes Dev. 2003, 17, 354-358. [CrossRef] [PubMed]

8. Koizumi, K.; Wu, S.; MacRae-Crerar, A.; Gallagher, K.L. An essential protein that interacts with endosomes and promotes movement of the short-root transcription factor. Curr. Biol. 2011, 21, 1559-1564. [CrossRef] [PubMed]

9. Clark, N.M.; Hinde, E.; Winter, C.M.; Fisher, A.P.; Crosti, G.; Blilou, I.; Gratton, E.; Benfey, P.N.; Sozzani, R. Tracking transcription factor mobility and interaction in Arabidopsis roots with fluorescence correlation spectroscopy. Elife 2016, 5, e14770. [CrossRef] [PubMed]

10. Koizumi, K.; Hayashi, T.; Gallagher, K.L. SCARECROW reinforces short-root signaling and inhibits periclinal cell divisions in the ground tissue by maintaining SHR at high levels in the endodermis. Plant Signal. Behav. 2012, 7, 1573-1577. [CrossRef] [PubMed]

11. Nakajima, K.; Sena, G.; Nawy, T.; Benfey, P.N. Intercellular movement of the putative transcription factor SHR in root patterning. Nature 2001, 413, 307-311. [CrossRef] [PubMed]

12. Della Rovere, F.; Fattorini, L.; D’Angeli, S.; Veloccia, A.; Del Duca, S.; Cai, G.; Falasca, G.; Altamura, M.M. Arabidopsis SHR and SCR transcription factors and AUX1 auxin influx carrier control the switch between adventitious rooting and xylogenesis in planta and in in vitro cultured thin cell layers. Ann. Bot. 2015, 115, 617-628. [CrossRef] [PubMed]

13. Ogasawara, H.; Kaimi, R.; Colasanti, J.; Kozaki, A. Activity of transcription factor JACKDAW is essential for SHR/SCR-dependent activation of SCARECROW and MAGPIE and is modulated by reciprocal interactions with MAGPIE, SCARECROW and SHORT ROOT. Plant Mol. Biol. 2011, 77, 489-499. [CrossRef] [PubMed] 
14. Hirano, Y.; Nakagawa, M.; Suyama, T.; Murase, K.; Shirakawa, M.; Takayama, S.; Sun, T.P.; Hakoshima, T. Structure of the SHR-SCR heterodimer bound to the BIRD/IDD transcriptional factor JKD. Nat. Plants 2017, 3, 17010. [CrossRef] [PubMed]

15. Carlsbecker, A.; Lee, J.-Y.; Roberts, C.J.; Dettmer, J.; Lehesranta, S.; Zhou, J.; Lindgren, O.; Moreno-Risueno, M.A.; Vatén, A.; Thitamadee, S. Cell signalling by microRNA165/6 directs gene dose-dependent root cell fate. Nature 2010, 465, 316-321. [CrossRef] [PubMed]

16. Scheres, B.; Di Laurenzio, L.; Willemsen, V.; Hauser, M.T.; Janmaat, K.; Weisbeek, P.; Benfey, P.N. Mutations affecting the radial organisation of the Arabidopsis root display specific defects throughout the embryonic axis. Development 1995, 121, 53-62.

17. Wysocka-Diller, J.W.; Helariutta, Y.; Fukaki, H.; Malamy, J.E.; Benfey, P.N. Molecular analysis of SCARECROW function reveals a radial patterning mechanism common to root and shoot. Development 2000, 127, 595-603. [PubMed]

18. Ueda, M.; Koshino-Kimura, Y.; Okada, K. Stepwise understanding of root development. Curr. Opin. Plant Biol. 2005, 8, 71-76. [CrossRef] [PubMed]

19. Qi, B.; Yang, Y.; Yin, Y.; Xu, M.; Li, H. De novo sequencing, assembly, and analysis of the Taxodium 'zhongshansa' roots and shoots transcriptome in response to short-term waterlogging. BMC Plant Biol. 2014, 14, 1. [CrossRef] [PubMed]

20. Yu, C.; Xu, S.; Yin, Y. Transcriptome analysis of the Taxodium 'zhongshanshan 405'roots in response to salinity stress. Plant Physiol. Biochem. 2016, 100, 156-165. [CrossRef] [PubMed]

21. Tamura, K.; Peterson, D.; Peterson, N.; Stecher, G.; Nei, M.; Kumar, S. MEGA5: Molecular evolutionary genetics analysis using maximum likelihood, evolutionary distance, and maximum parsimony methods. Mol. Biol. Evol. 2011, 28, 2731-2739. [CrossRef] [PubMed]

22. Wang, Z.; Gu, C.; Xuan, L.; Hua, J.; Shi, Q.; Fan, W.; Yin, Y.; Yu, F. Identification of suitable reference genes in Taxodium 'Zhongshanshan' under abiotic stresses. Trees 2017, 1-12. [CrossRef]

23. Xu, M.; Zhang, B.; Su, X.; Zhang, S.; Huang, M. Reference gene selection for quantitative real-time polymerase chain reaction in Populus. Anal. Biochem. 2011, 408, 337-339. [CrossRef] [PubMed]

24. Xu, M.; Xie, W.; Huang, M. Overexpression of PeRHD3 alters the root architecture in Populus. Biochem. Biophys. Res. Commun. 2012, 424, 239-244. [CrossRef] [PubMed]

25. Tan, B.; Xu, M.; Chen, Y.; Huang, M. Transient expression for functional gene analysis using Populus protoplasts. Plant Cell Tissue Organ Cult. 2013, 114, 11-18. [CrossRef]

26. Combet, C.; Blanchet, C.; Geourjon, C.; Deléage, G. NPS@: Network protein sequence analysis. Trends Biochem. Sci. 2000, 25, 147-150. [CrossRef]

27. Sigrist, C.J.A.; De Castro, E.; Cerutti, L.; Cuche, B.A.; Hulo, N.; Bridge, A.; Bougueleret, L.; Xenarios, I. New and continuing developments at PROSITE. Nucleic Acids Res. 2012, 41, D344-D347. [CrossRef] [PubMed]

28. Zhang, D.; Iyer, L.M.; Aravind, L. Bacterial GRAS domain proteins throw new light on gibberellic acid response mechanisms. Bioinformatics 2012, 28, 2407-2411. [CrossRef] [PubMed]

29. Cenci, A.; Rouard, M. Evolutionary Analyses of GRAS Transcription Factors in Angiosperms. Front. Plant Sci. 2017, 8. [CrossRef] [PubMed]

30. Kamiya, N.; Itoh, J.I.; Morikami, A.; Nagato, Y.; Matsuoka, M. The SCARECROW gene's role in asymmetric cell divisions in rice plants. Plant J. 2003, 36, 45-54. [CrossRef] [PubMed]

31. Xuan, L.; Xu, M.; Chen, C.; Yang, C.; Xu, L.; Huang, M. Identification and characterization of three PeSHRs and one PeSCR involved in adventitious root development of Populus. Plant Cell Tissue Organ Cult. 2014, 117, 253-264. [CrossRef]

32. Bolle, C. The role of GRAS proteins in plant signal transduction and development. Planta 2004, 218, 683-692. [CrossRef] [PubMed]

33. Yoon, E.K.; Dhar, S.; Lee, M.-H.; Song, J.H.; Lee, S.A.; Kim, G.; Jang, S.; Choi, J.W.; Choe, J.-E.; Kim, J.H. Conservation and diversification of the SHR-SCR-SCl23 regulatory network in the development of the functional endodermis in Arabidopsis shoots. Mol. Plant 2016, 9, 1197-1209. [CrossRef] [PubMed]

34. Kaufmann, K.; Pajoro, A.; Angenent, G.C. Regulation of transcription in plants: Mechanisms controlling developmental switches. Nat. Rev. Genet. 2010, 11, 830-842. [CrossRef] [PubMed]

35. Pysh, L.D.; Wysocka-Diller, J.W.; Camilleri, C.; Bouchez, D.; Benfey, P.N. The GRAS gene family in Arabidopsis: Sequence characterization and basic expression analysis of the SCARECROW-like genes. Plant J. 1999, 18, 111-119. [CrossRef] [PubMed] 
36. Lee, M.-H.; Kim, B.; Song, S.-K.; Heo, J.-O.; Yu, N.-I.; Lee, S.A.; Kim, M.; Kim, D.G.; Sohn, S.O.; Lim, C.E. Large-scale analysis of the GRAS gene family in Arabidopsis thaliana. Plant Mol. Biol. 2008, 67, 659-670. [CrossRef] [PubMed]

37. Helariutta, Y.; Fukaki, H.; Wysocka-Diller, J.; Nakajima, K.; Jung, J.; Sena, G.; Hauser, M.-T.; Benfey, P.N. The short-root gene controls radial patterning of the Arabidopsis root through radial signaling. Cell 2000, 101, 555-567. [CrossRef]

38. Wang, J.; Andersson-Gunnerås, S.; Gaboreanu, I.; Hertzberg, M.; Tucker, M.R.; Zheng, B.; Leśniewska, J.; Mellerowicz, E.J.; Laux, T.; Sandberg, G. Reduced expression of the short-root gene increases the rates of growth and development in hybrid poplar and Arabidopsis. PLoS ONE 2011, 6, e28878. [CrossRef] [PubMed]

39. Wu, S.; Gallagher, K.L. Intact microtubules are required for the intercellular movement of the short-root transcription factor. Plant J. 2013, 74, 148-159. [CrossRef] [PubMed]

40. Lim, J.; Helariutta, Y.; Specht, C.D.; Jung, J.; Sims, L.; Bruce, W.B.; Diehn, S.; Benfey, P.N. Molecular analysis of the SCARECROW gene in maize reveals a common basis for radial patterning in diverse meristems. Plant Cell 2000, 12, 1307-1318. [CrossRef] [PubMed]

41. Richards, D.E.; Peng, J.; Harberd, N.P. Plant GRAS and metazoan STATs: One family? Bioessays 2000, 22, 573-577. [CrossRef]

42. Miyashima, S.; Hashimoto, T.; Nakajima, K. ARGONAUTE1 acts in Arabidopsis root radial pattern formation independently of the SHR/SCR pathway. Plant Cell Physiol. 2009, 50, 626-634. [CrossRef] [PubMed]

43. Heidstra, R.; Welch, D.; Scheres, B. Mosaic analyses using marked activation and deletion clones dissect Arabidopsis SCARECROW action in asymmetric cell division. Genes Dev. 2004, 18, 1964-1969. [CrossRef] [PubMed]

(C) 2017 by the authors. Licensee MDPI, Basel, Switzerland. This article is an open access article distributed under the terms and conditions of the Creative Commons Attribution (CC BY) license (http:/ / creativecommons.org/licenses/by/4.0/). 